\title{
Prediction of a geosynchronous electron environment with in situ magnetic field measurements
}

\author{
A. Tsutai, C. Mitsui, and T. Nagai \\ Earth and Planetary Sciences, Tokyo Institute of Technology, Tokyo 152-8551, Japan
}

(Received August 20, 1998; Revised December 28, 1998; Accepted December 28, 1998)

\begin{abstract}
A new approach to predict the relativistic electron flux at geosynchronous orbit has been developed. Our prediction technique is based on a linear prediction filter with the input consisting of magnetic field variations at geosynchronous orbit for 6 consecutive days. The output is a prediction, made one day ahead, of the average daily flux of $>2 \mathrm{MeV}$ electrons. This technique was developed with data from GOES 7 for the period from January 1994 through April 1994. The technique's performance was tested by comparing the output results with the fluxes observed for the period from May 1994 through May 1995. The results showed that the empirical technique using in situ magnetic field measurements provides reasonably accurate forecasts of the geosynchronous electron environment one day in advance.
\end{abstract}

\section{Introduction}

One of the major goals of space weather research is to predict the flux of relativistic $(>1 \mathrm{MeV}$ ) electrons at geosynchronous orbit. This prediction is important because these electrons can have a serious impact on space systems (e.g., Hastings and Garrett, 1996). Nagai (1988) has shown that a linear prediction filtering technique has considerable promise for practical forecasting. Using this technique, Nagai reproduced the logarithm of the daily average flux (electrons $/ \mathrm{cm}^{2}$ s-ster) of $>2 \mathrm{MeV}$ electrons (log EL) observed at the geostationary satellite GMS-3. As input, he used the daily sums of $K p(\Sigma K p)$ for 10 consecutive days. Baker et al. (1990) critically tested this method using the extended data sets. They noted that (1) the relationship between $\Sigma K p$ and log EL is not time stationary, (2) the relationship is not linear, and (3) there are probably other factors that cause the electron flux to increase. However, they confirmed that event timing can be predicted well using $\Sigma K p$. Baker et al. (1990) made a prediction filter using data from the period 1982-1985 and made predictions for the 6-month period July-December 1984. However, a prediction filter can be produced even with data for a much shorter period. Since we can update a prediction filter, problem (1) - the fact that the relationship is not time stationary-is not serious. Koons and Gorney (1991) made a neural network model and showed that its results are significantly more accurate than the results from linear prediction filters. Hence, problem (2) (nonlinearity) can be solved. As for problem (3), although there might be electron flux increases that are not associated with geomagnetic activity, they are not common. One practical problem for the prediction of the electron flux is that we cannot obtain $\Sigma K p$ for the immediate past 10 days.

The current NOAA/GOES geosynchronous satellites have

Copy right (C) The Society of Geomagnetism and Earth, Planetary and Space Sciences (SGEPSS); The Seismological Society of Japan; The Volcanological Society of Japan; The Geodetic Society of Japan; The Japanese Society for Planetary Sciences. the capability of measuring the magnetic field. Variations in the magnetic field at synchronous orbit are strongly related to substorm activity (e.g., Nagai, 1982a,b). Although the mechanism for electron flux increases is not fully understood, it is likely that the increases are well correlated with substorm and storm activity (e.g., Baker et al., 1986; Nagai, 1988; Li et al., 1997). In this paper, we propose a new input parameter that is based on measurements of the magnetic field at geosynchronous orbit. To test the applicability of our new method, we use a linear prediction filter technique.

\section{Methodology}

We have used the data obtained by GOES 7 for the period from January 1994 through April 1995. GOES 7 was located at $112^{\circ} \mathrm{W}$, and its magnetic latitude was $10^{\circ}$. Daily average values of the electron $(>2 \mathrm{MeV})$ flux are determined using 5-minute average values. For an input parameter, the 5-minute average values of the magnetic field $H p$ are used. $H p$ is perpendicular to the spacecraft orbital plane, and is approximately the northward component of the magnetic field. Since the $H p$ field corresponds to the $H$ component in the usual VDH coordinate system, substorm-associated variations are easily recognized. The northward magnetic field decreases prior to the onset of a substorm, and it returns to or exceeds the quiet-day value in association with the onset. Typically, these magnetic variations have time scales of less than one hour (e.g., Nagai, 1991). The magnitude of the change in the northward magnetic field during substorms is well correlated with substorm magnitude (e.g., Kokubun and McPherron, 1981).

The index for magnetic variations at geosynchronous orbit (MAG) is derived as follows. First, the diurnal variation of the $H p$ field for each day is obtained by discarding variations with time scales shorter than 1.5 hours. For each day, a data set originally consists of 288 data points. To the front of this data set are added 112 data points from the period 1440-2400 UT of the previous day, and to the rear of this data set are 


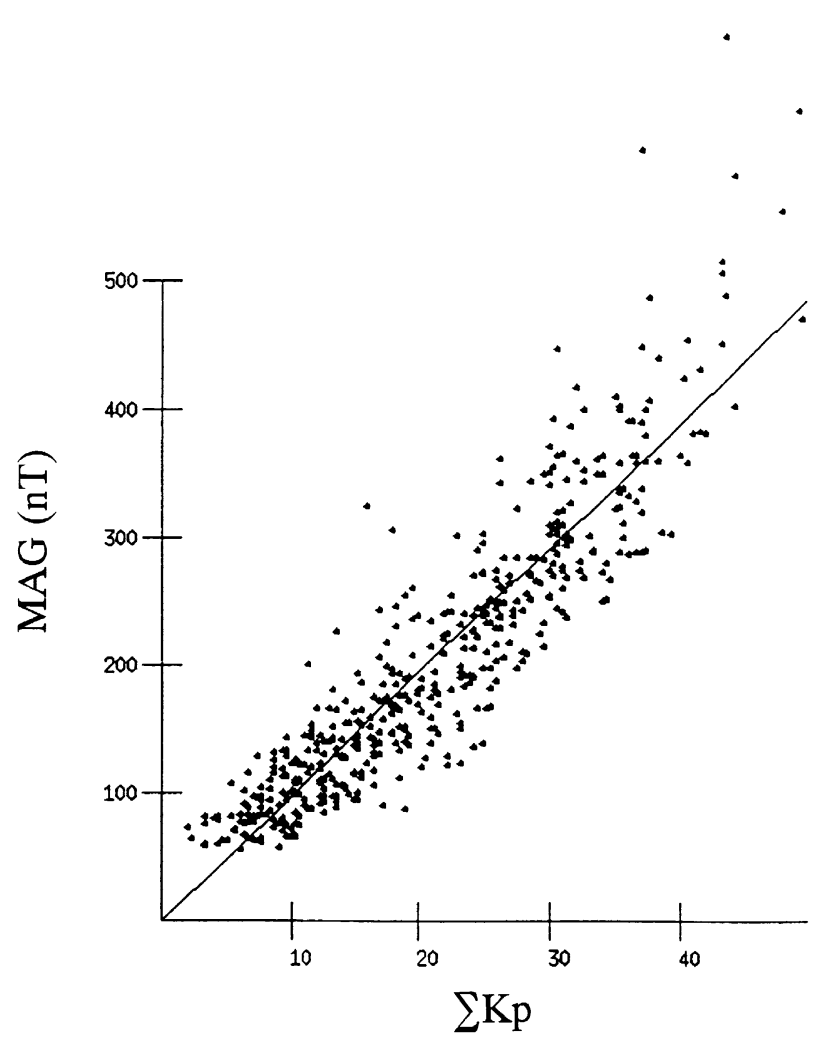

Fig. 1. Scatter plot of MAG (magnitudes of perturbations at geosynchronous orbit, see the text) versus $\Sigma K p$. The line indicates the linear regression relationship.

added 112 data points from the period 0000-0920 UT (the difference between the point at 2400 UT and that at 0000 UT is adjusted). Using the data set of 512 data points, we perform a fast Fourier transform analysis and then obtain the diurnal variation.

Next, we calculate the magnitude of the change in $\mathrm{Hp}$ for each of 47 one-hour periods (with 30-minute overlapping), after subtracting the diurnal variation from the original $\mathrm{Hp}$ values. Finally, we sum up these 47 values to obtain MAG (in units of nT).

Figure 1 shows a scatter plot of MAG versus $\Sigma K p$ for the period from January 1994 through April 1995. The correlation is very high; the correlation coefficient is 0.91 (the line indicates the linear regression relationship). Although MAG is produced with one-point observations, it reasonably represents global-scale geomagnetic activity.

Figure 2 shows the impulse response functions computed from the $\Sigma K p$ input time series and the MAG input time series. The input time series cover the period from January through April 1994. The output is log EL. The procedure for calculating the impulse response function is the same as that described by Nagai (1988). These two functions show very similar impulse responses, which increase strongly, peak at a lag of 2 days, and show small contributions beyond a lag of 4 days. The impulse response function obtained by Nagai (1988) shows a decrease at zero lag (see also Baker et al., 1990). In this period, there was a strong recurrence of geomagnetic activity; and the electron flux showed a sharp increase followed by a gradual decrease for each enhance-
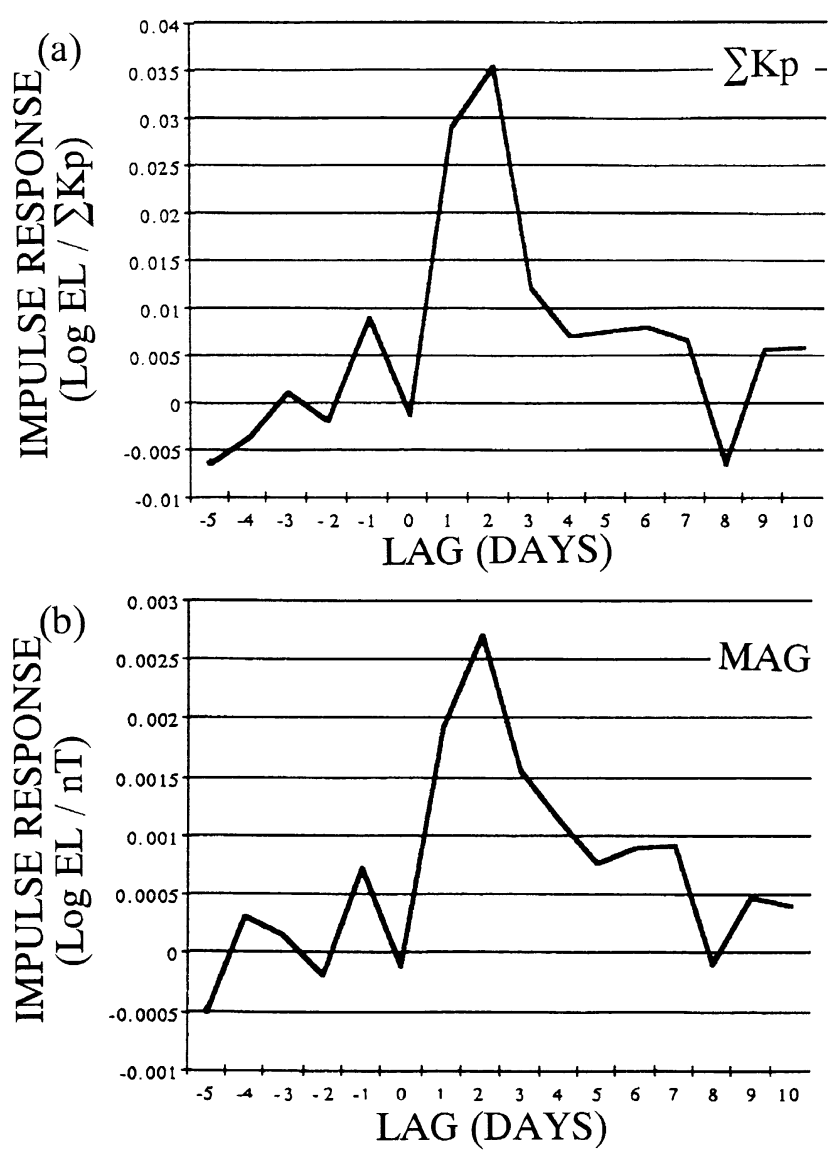

Fig. 2. Impulse response functions computed for the input time series of $\Sigma K p$ (a) and for the input time series of MAG (b).

ment in geomagnetic activity. Hence, this impulse response function may not be suitable for periods of non-recurrent geomagnetic activity.

\section{Prediction}

In order to evaluate how well we can "predict" the electron flux, we "forecast" the electron flux (log EL) one day ahead. In Nagai (1988), input was constructed with data for consecutive 10 days. We tested prediction skill for different data sets. The prediction skill does not change significantly for the input constructed with the data for more than 4 day, and rms errors have a small minimum for the input data for 6 days. Here, we use 6-day input sets of MAG and $\Sigma K p$. Figure 3 presents the input data and output results for the period from January through April 1994. The heavy curves show predicted values, whereas the thin curves show observed values. Since the impulse response functions are calculated with the same data sets, the results are not a true "prediction". In this "in-sample analysis", we have found that the 6-day input sets of MAG or $\Sigma K p$ are reasonable in predicting the flux.

The true "prediction" results are presented in Figs. 4-6 for the period from May 1994 through April 1995, whereas the impulse response functions are calculated with data for the period from January through April 1994. We do not get perfect agreement between the predicted and observed electron 


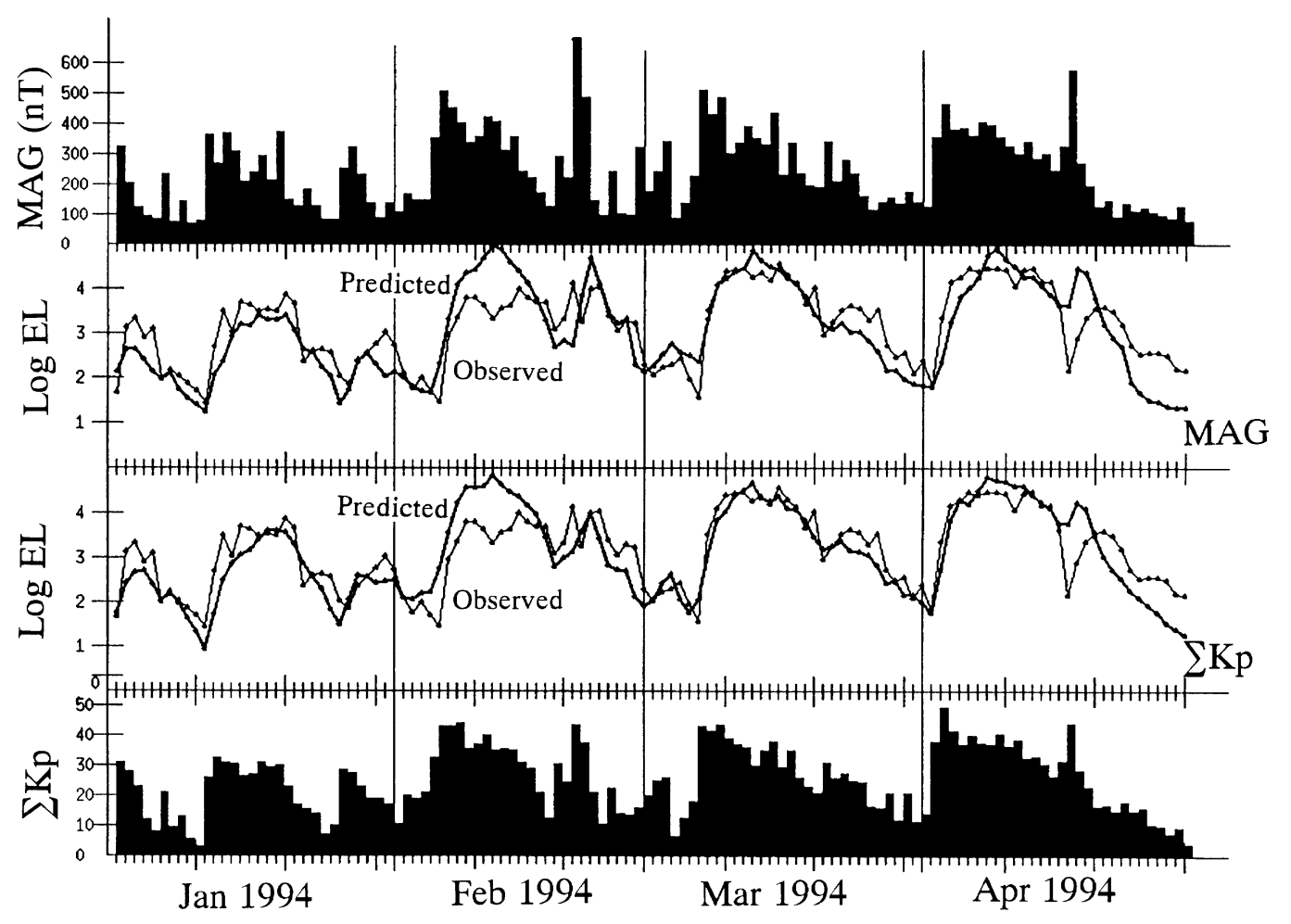

Fig. 3. Predicted values and observed values of electron flux (log EL) for the period from January 1994 through April 1994. The second panel presents the results from MAG, whereas the third panel shows results from $\Sigma K p$. MAG and $\Sigma K p$ are also plotted.

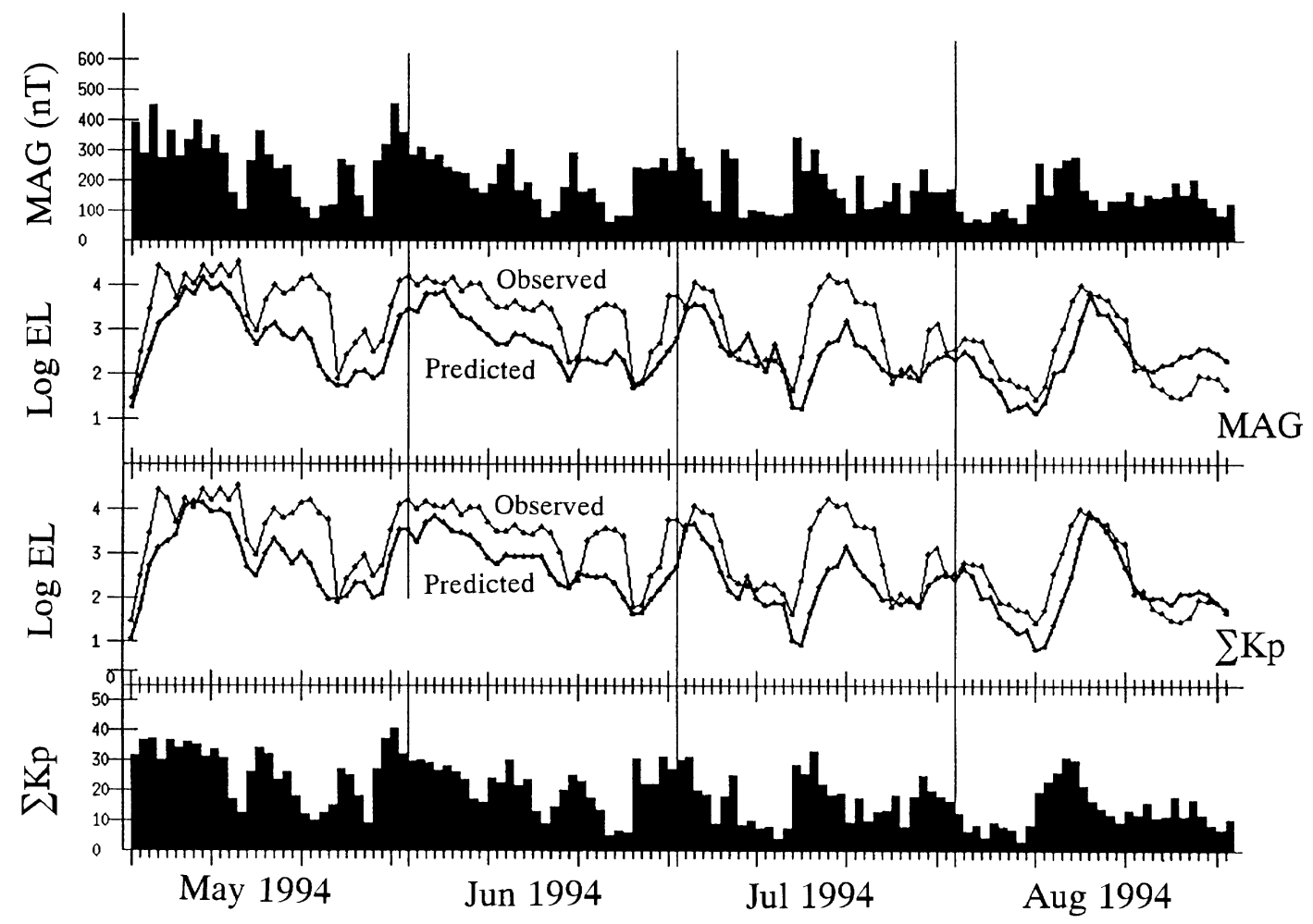

Fig. 4. Predicted values and observed values of electron flux (log EL) for the period from May 1994 through August 1994 . The second panel presents the results from MAG, whereas the third panel shows results from $\Sigma K p$. MAG and $\Sigma K p$ are also plotted. 


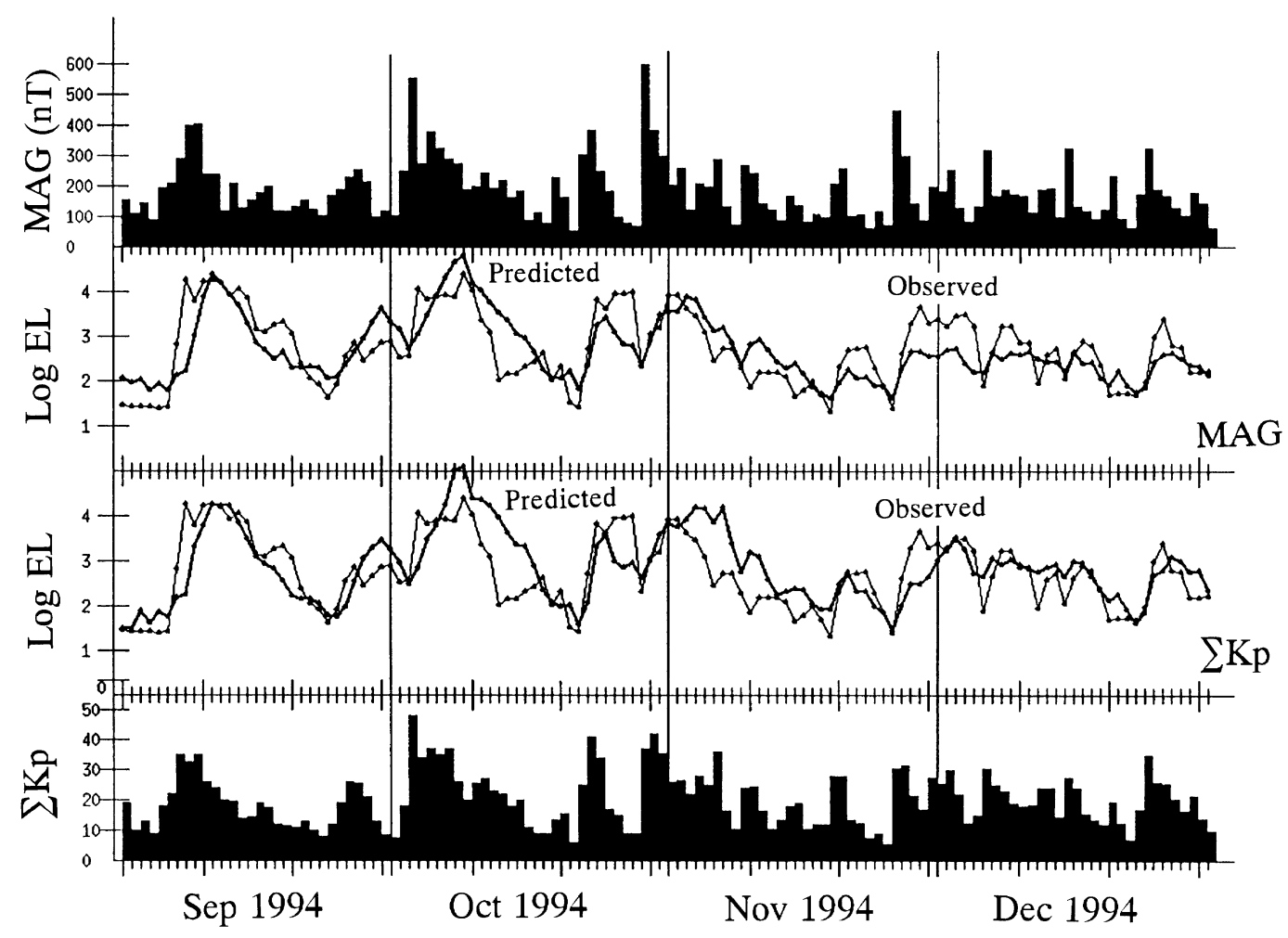

Fig. 5. Predicted values and observed values of electron flux (log EL) for the period from September 1994 through December 1994 . The second panel presents the results from MAG, whereas the third panel shows results from $\Sigma K p$. MAG and $\Sigma K p$ are also plotted.

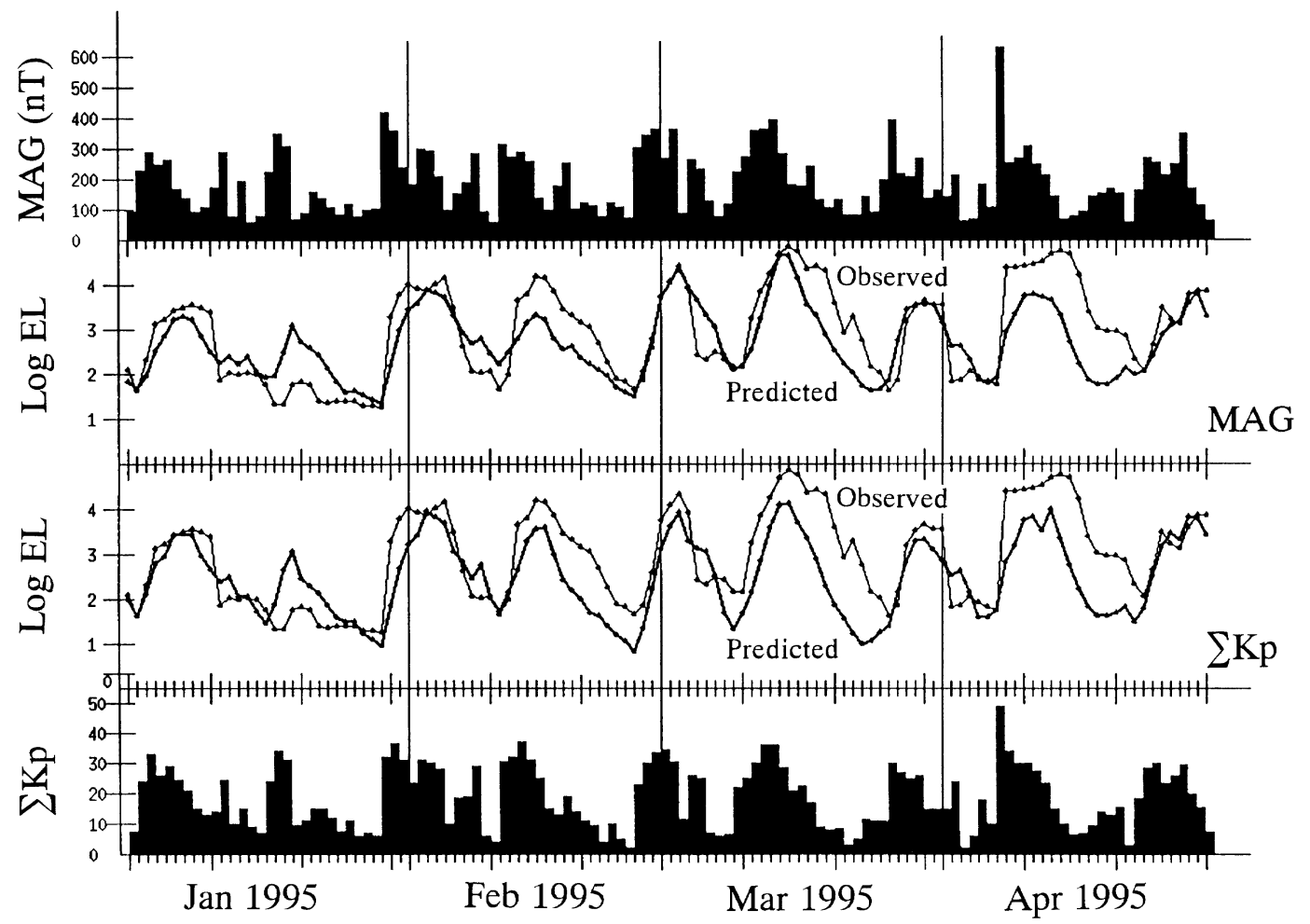

Fig. 6. Predicted values and observed values of electron flux (log EL) for the period from January 1995 through April 1995 . The second panel presents the results from MAG, whereas the third panel shows results from $\Sigma K p$. MAG and $\Sigma K p$ are also plotted. 
fluxes in this "out-of-sample analysis". The rms error of the analysis with MAG is 0.68 , while the rms error of the analysis with $\Sigma K p$ is 0.73 . However, the predicted values trace the observed values well. It is especially noteworthy that all of the increases in the electron flux are successfully predicted. In May-July 1994, the predicted values are generally lower than the observed values; however, the values predicted with MAG are very similar to those predicted with $\Sigma K p$. The predicted values reach the observed values in August-October 1994. Hence, the discrepancy seen in May-July 1994 is presumably caused by the linear prediction technique rather than the input data. Increases in the predicted values are sometimes delayed with respect to the observed values, as seen in the data for July-August 1994.

\section{Discussion and Conclusions}

We have proposed a practical technique for predicting the electron flux at geosynchronous orbit. The technique is basically the same as that described by Nagai (1988), who used 10 consecutive days of $\Sigma K p$. Practically, however, we cannot obtain $\Sigma K p$ for yesterday. With the new technique, input data are produced using magnetic field measurements at synchronous orbit for the past 6 days. The technique provides a reasonably good prediction of the geosynchronous electron environment one day in advance.

It is important, however, to note limitations in the usage of the proposed method. As indicated in Section 2, the impulsive response function obtained for the data in 1994 is different from that obtained for the data in 1984-1985 (Nagai, 1988). We have to investigate representative responses of the electron flux to various types of geomagnetic activity, i.e., recurrent geomagnetic activity, non-recurrent geomagnetic activity, and continuous high geomagnetic activity. These studies would provide information on updating of prediction functions. Furthermore, the linear prediction filter technique has larger errors for higher electron fluxes, as indicated by
Koons and Gorney (1991). Practically, it is important to make a good prediction for higher electron fluxes (e.g., higher than $10^{3}$ electrons $/ \mathrm{cm}^{2}$-s-ster). Since a neural network model can maintain its good skill in prediction over the higher range of electron fluxes, highly sophisticated methods (e.g., neural network models) should be tested.

Acknowledgments. The GOES particle and magnetic field data were provided by the National Geophysical Data Center, NOAA.

\section{References}

Baker, D. N., J. B. Blake, R. W. Klebesadel, and P. R. Higbie, Highly relativistic electrons in the Earth's outer magnetosphere, 1. Lifetimes and temporal history 1979-1984, J. Geophys. Res., 91, 4265-4276, 1986.

Baker, D. N., R. L. McPherron, T. E. Cayton, and R. W. Klebesadel, Linear prediction filter analysis of relativistic electron properties at $6.6 R_{E}, J$. Geophys. Res., 95, 15133-15140, 1990.

Hastings, D. and H. Garrett, Spacecraft-Environment Interactions, 292 pp., Cambridge University Press, New York, 1996.

Kokubun, S. and R. L. McPherron, Substorm signatures at synchronous altitude, J. Geophys. Res., 86, 11265-11277, 1981.

Koons, H. C. and D. J. Gorney, A neural network model of the relativistic electron flux at geosynchronous orbit, J. Geophys. Res., 96, 5549-5556, 1991.

Li, X., D. N. Baker, M. Temerin, D. Larson, R. P. Lin, G. D. Leeves, M. Looper, S. G. Kanekal, and R. A. Mewaldt, Are energetic electrons in the solar wind the source of the outer radiation belt?, Geophys. Res. Lett., 24, 923-926, 1997.

Nagai, T., Local time dependence of electron flux changes during substorms derived from multi-satellite observations at synchronous orbit, J. Geophys. Res., 87, 3456-3468, 1982a.

Nagai, T., Observed magnetic substorm signatures at synchronous altitude, J. Geophys. Res., 87, 4405-4417, 1982b.

Nagai, T., "Space weather forecast": Prediction of relativistic electron intensity at synchronous orbit, Geophys. Res. Lett., 15, 425-428, 1988.

Nagai, T., An empirical model of substorm-related magnetic field variations at synchronous orbit, in Magnetospheric Substorms, edited by J. R. Kan, T. A. Potemra, S. Kokubun, and T. Iijima, pp. 91-95, AGU, Washington, D.C., 1991.

A. Tsutai, C. Mitsui, and T. Nagai (e-mail: nagai@geo.titech.ac.jp) 